\title{
EVALUACIÓN DEL ESTADO NUTRICIONAL DE MUJERES GESTANTES QUE PARTICIPARON DE UN PROGRAMA DE ALIMENTACIÓN Y NUTRICIÓN
}

\author{
ASSESSMENT OF NUTRITIONAL STATUS \\ OF A GROUP OF PREGNANT WOMEN WHO \\ PARTICIPATED IN A FOOD AND NUTRITION PROGRAM
}

\begin{abstract}
Sandra Lucía Restrepo M., Lorena Patricia Mancilla L., Beatriz Elena Parra S., Luz Mariela Manjarrés C. Natalia Janeth Zapata L., Paula Andrea Restrepo Ochoa., Mónica Isabel Martínez S.

Escuela de Nutrición y Dietética, Universidad de Antioquia, Colombia.
\end{abstract}

\begin{abstract}
Objective: To evaluate the nutritional status of a group of pregnant women and their newborn infants participating in the MANA program. Methods: Descriptive, longitudinal and prospective study designed to follow the cohort before and after intervention. Results: $53 \%$ of the household showed food insecurity. With the program food ingestion had a significant increase and the prevalence of the risk of micronutrient deficiency diminished. The low gestational weight diminished from $27.8 \%$ in the first trimester to $20.3 \%$ in the third trimester. $94 \%$ of the newborns weighed over $2500 \mathrm{~g}$. Anemia was prevented in $86 \%$ of the mothers with iron deficiency. The mothers at risk of serum folate deficiency diminished from $30,8 \%$ in the first trimester to $11,5 \%$ in the second. Conclusion: Since the socioeconomic conditions of the population were unchanged during the study, it is possible to conclude that the MANA program and the products received had a positive and significant impact in the nutritional status of the mothers.
\end{abstract}

Key words: Pregnancy; food complementation; micronutrients supplementation; nutritional education; nutritional status.

Este trabajo fue recibido el 17 de Marzo de 2009 y aceptado para ser publicado el 12 de Diciembre de 2009.

\section{INTRODUCCIÓN}

La mortalidad materna constituye en la actualidad uno de los principales problemas de salud pública que afronta el país. El informe de la Situación de Salud en Colombia 2007, reportó una tasa de 73 por 100 mil nacidos vivos[1] y el departamento de Antioquia también presenta una situación preocupante ya que en el año 2006 registró una tasa de 56,8 por 100 mil nacidos vivos [2]. La mortalidad y morbilidad materna son problemas multicausales asociados a situaciones complejas y diversas, entre ellas alimentarias y nutricionales, determinadas por el incremento de calorías y nutrientes en la madre para mantener sus procesos vitales y los del futuro hijo.

La Encuesta Nacional de la Situación Nutricional 2005 reportó que en Colombia 52,4\% de las gestantes entre 13 y 17 años, $41,3 \%$ entre 18 y 29 años y 48,2\% entre 30 y 49 años, tenían anemia. Esta situación se acompaña de un inadecuado estado nutricional, ya que en el país 5 de cada 10 gestantes sufren malnutrición y de éstas $40 \%$ tienen déficit de peso, lo que puede incidir en el bajo peso al nacer (BPN) que a nivel nacional alcanza el $6.2 \%$ [3] .

El déficit nutricional, el bajo peso pregestacional y la inadecuada ganancia de peso en la gestación, incrementan el riesgo de insuficiencia cardiaca para la madre y el feto, parto prematuro, defectos del tubo neural y bajo peso al nacer [4] a este último le genera en etapas posteriores de la vida consecuencias deletéreas como trastornos del aprendizaje, alteraciones en el desarrollo psicomotor y de crecimiento y mayor riesgo de padecer enfermedades crónicas en la edad adulta $[4,5]$.

Por todo lo anterior, la atención a la mujer gestante requiere de una cuidadosa vigilancia alimentaria y nutricional que permita la detección oportuna de riesgo 
y el desarrollo de intervenciones que contribuyan a la salud del binomio madre hijo. En el caso colombiano la resolución 412 del 2000 [6] contempla las actividades de atención obligatoria para este grupo de población en las cuales se incluyen las de alimentación y nutrición, sin embargo se evidencia baja calidad en la atención, que no contribuye al buen desarrollo del proceso gestacional [7] .

Por todo lo anterior y ante la carencia de estudios en nuestro medio que den cuenta de los resultados de intervenciones alimentarias y nutricionales en este grupo de población, se desarrolló este proyecto, el cual tuvo como propósito evaluar el estado nutricional de un grupo de gestantes que participaron en un programa de intervención nutricional que incluyó complementación alimentaria, suplemento de micronutrientes, monitoreo de la ganancia de peso y educación nutricional.

\section{SUJETOS Y MÉTODOS}

Se realizó un estudio descriptivo, longitudinal y prospectivo [8] con seguimiento de la cohorte de gestantes seleccionadas, antes y después de una intervención nutricional. Este estudio se considera una evaluación expost, ya que valoró los efectos generados en las gestantes una vez finalizada la intervención nutricional [9].

En el desarrollo del proyecto se consideraron variables socioeconómicas (edad, lugar de residencia, escolaridad, ocupación, jefatura del hogar, ingresos familiares, materiales y condiciones de la vivienda y percepción de la seguridad alimentaria), de ingesta dietética (ingesta de calorías, macro y micronutrientes), antropométricas (peso pregestacional, índice de masa corporal, ganancia de peso gestacional, peso del recién nacido) y bioquímicas (hemoglobina, hematocrito, volumen corpuscular medio, ferritina, folato sérico).

La población de referencia estuvo constituida por 2.000 gestantes participantes del programa de Mejoramientos Alimentario y Nutricional para Antioquia (MANA) para la vida en 30 municipios de las subregiones de Bajo Cauca, Norte, Urabá (Antioquia - Colombia). Ante las dificultades para lograr una muestra representativa que cumpliera con los criterios de inclusión requeridos para el desarrollo del proyecto, fue necesario establecer una muestra por conveniencia de 105 gestantes seleccionadas entre voluntarias que fueron a consulta médica a los diferentes hospitales en los cuales se desarrolló el programa (figura 1) y que cumplieron con los siguientes criterios de inclusión: edad entre 16 y 40 años, ingreso al programa prenatal antes de la semana 13 de gestación, mujeres sanas, con embarazo de feto único, que no estuvieran participando de programas de complementación alimentaria y/o consumiendo suplementos de micronutrientes.

Por los altos costos de las pruebas bioquímicas, la necesidad de equipos especializados para su procesamiento y las dificultades en el desplazamiento de las mismas de las regiones a los laboratorios donde serían procesadas, fue necesario seleccionar una de las regiones para el desarrollo del componente bioquímico. De acuerdo con lo anterior, se eligió la región del Bajo Cauca en la cual se evaluaron un total de 26 gestantes, a las cuales además de la aplicación de la encuesta sociodemográfica y de seguridad alimentaria, la evaluación de ingesta dietética y antropométrica, se les realizó la evaluación bioquímica.

El programa de intervención consistió en la entrega de un complemento alimentario, un suplementos de micronutrientes, monitoreo de la ganancia de peso y educación nutricional. El complemento alimentario estuvo compuesto por leche en polvo, galletas y bienestarina $^{1}$ y aportó 300 calorías/día. La leche en polvo diseñada especialmente para la intervención aportó el $60 \%$ de los requerimientos diarios de vitaminas A, D3, E, B1, B2, B3, B6, B12, C, ácido fólico y minerales como calcio y cinc. El suplemento de micronutrientes aportó 60 miligramos de hierro elemental en forma de fumarato ferroso, 400 microgramos de ácido fólico y 70 miligramos de vitamina C. la entrega de estos productos se acompañó de un monitoreo de la ganancia de peso y educación nutricional.

El proceso educativo, se desarrolló en cada región de acuerdo con el diagnóstico y las necesidades en educación alimentaria y nutricional de las gestantes. Para el desarrollo de las actividades se utilizó como estrategia metodológica el compartir de saberes y los talleres de preparación de alimentos en pequeños grupos. Las madres recibieron capacitación en temáticas como: importancia de la alimentación y nutrición para la salud de la madre y del bebé en formación, preparación y adecuado consumo del complemento alimentario y la importancia del consumo y adherencia al suplemento de micronutrientes.

La capacitación se acompañó de un cuadernillo en el cual la madre profundizaba en las actividades realizadas en su casa en compañía de otros miembros de la familia. Las sesiones educativas estuvieron a cargo de profesionales de la salud encargados de los programas de control prenatal, a los cuales se les hizo una capacitación y estandarización previa.

Para la recolección de los datos se precisaron los

1. La bienestariana es un alimento en forma de harina, compuesta en un $30 \%$ de harina de leguminosa que por lo general es soya desgrasada, $70 \%$ harina de cereal (mezcla de cereales), $8 \%$ leche en polvo entera y $2 \%$ de una premezcla de vitaminas y minerales. 
métodos que garantizaran la calidad de los mismos y se hizo la selección de un grupo de profesionales con experiencia en este tipo de proyectos. Dicha recolección estuvo a cargo de 6 nutricionistas dietistas ( 2 por región) quienes previamente tuvieron 100 horas de capacitación y estandarización y que evaluaron a las gestantes en cada trimestre (primer trimestre entre la semana 10 y14, en el segundo trimestre entre las semanas 23 y 25 y en el tercer trimestre después de la semana 35 ). Este grupo estuvo acompañado por los investigadores quienes realizaron vistas de campo para la supervisión en la recolección de datos.

\section{Procedimientos para la recolección de datos}

En el caso de los factores sociodemográficos y de seguridad alimentaria se aplicó una encuesta estructurada que contenía las preguntas correspondientes a los aspectos socioeconómicos del hogar, el consumo del suplemento de micronutrientes, del complemento alimentario y la escala de percepción de la seguridad alimentaria validada por Álvarez M [10] en un estudio realizado en el Departamento de Antioquia Colombia, y posteriormente aplicada en varios estudios a nivel nacional. La escala utilizada tiene varias ventajas ya que mide directamente el fenómeno de interés, es sencilla y de fácil diligenciamiento. Este instrumento se valora de acuerdo con un puntaje el cual clasifica la inseguridad alimentaria en: leve, moderada o severa.

Ingesta dietética: se realizaron dos recordatorios de 24 horas a cada gestante en cada uno de los trimestres de gestación.

La muestra estuvo distribuida a lo largo de la semana, y la segunda entrevista para evaluar la ingesta se realizó en días no consecutivos, este procedimiento permite ajustar la variabilidad intra e interindividual y obtener una información más aproximada de la adecua-

\section{FIGURA 1}

\section{Momentos del proyecto de investigación}

\begin{tabular}{l}
$\begin{array}{l}\text { PRIMER MOMENTO } \\
\text { Semanas } 10 \text { a } 13 \text { de } \\
\text { gestación }\end{array}$ \\
$\begin{array}{l}\text { Recolección de datos } \\
\text { demográficos, } \\
\text { socioeconómicos y de } \\
\text { salud. } \\
\text { Evaluación del estado } \\
\text { nutricional por medio de } \\
\text { indicadores } \\
\text { antropométricos } \\
\text { bioquimicos y } \\
\text { alimentarios. } \\
\text { Inicio del programa } \\
\text { MANA para la vida que } \\
\text { incluyó: } \\
\text { complementación } \\
\text { alimentaria, suplemento } \\
\text { de micronutrientes, } \\
\text { educación y vigilancia } \\
\text { nutricional }\end{array}$ \\
\hline
\end{tabular}

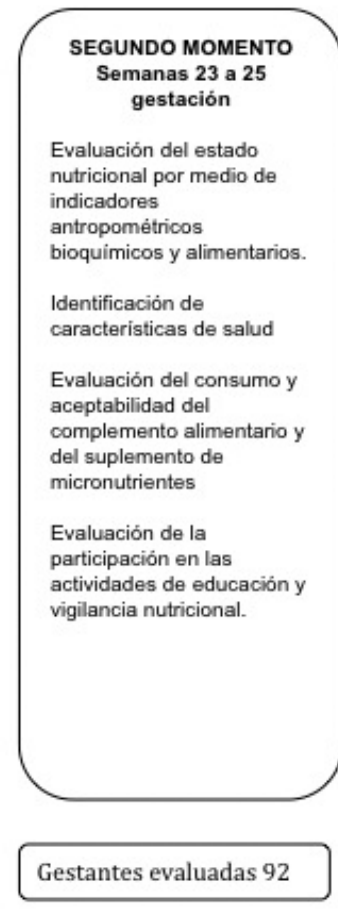

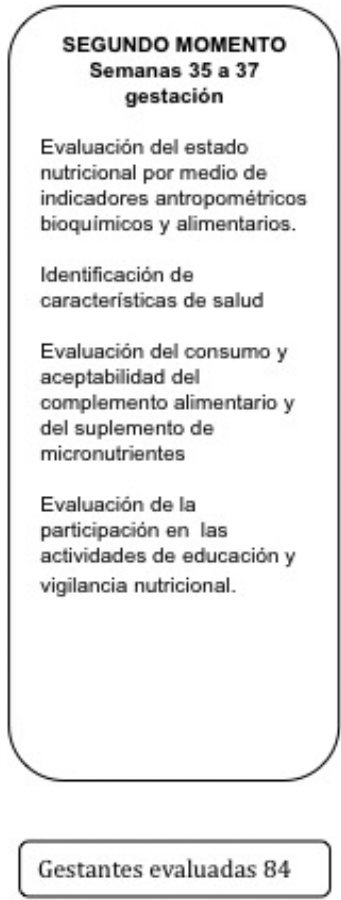

CUARTO MOMENTO

Nacimiento del bebé

Evaluación del peso y la longitud del recién nacido

Recién nacido evaluados 82 
ción del consumo de energía y nutrientes.

Las cantidades de los alimentos consumidos se precisaron con modelos, figuras geométricas y un álbum de fotografías con utensilios de medidas caseras en tamaño real, todos los instrumentos tenían el peso estandarizado de los productos que representaban.

Los recordatorios de 24 horas se ingresaron al Programa de Evaluación de la Ingesta Dietética de la Escuela de Nutrición de la Universidad de Antioquia [11] que tiene la información nutricional de las Tablas de Composición de Alimentos de Colombia [12], FAO - (Organización de las Naciones Unidas para la Agricultura y la Alimentación)- Latinfoods [13] y USDA Handbook 8 [14], este programa permite identificar la frecuencia en el consumo de alimentos, de energía y de nutrientes de cado uno de los recordatorios de 24 horas.

La información nutricional de cada uno de los recordatorios se procesó en el programa PC-SIDE (Personal Computer Version of Software for Intake Distribution Estimation), versión 1.0 de junio de 2004, disponible en el Departamento de Estadística de la Universidad del Estado de Iowa, Ames IA, USA, que ajusta la distribución de las ingestas considerando la variabilidad intra e interindividual y por ende una mejor aproximación a la prevalencia del riesgo de deficiencia del grupo de mujeres embarazadas en cada uno de los trimestres de gestación.

Para establecer la prevalencia del riesgo de deficiencia se consideraron las recomendaciones individuales de energía y de nutrientes, según edad de la materna, el trimestre de gestación y actividad física, los valores de referencia fueron los de las Dietary Reference Intakes (DRI) [15].

Indicadores antropométricos: La evaluación antropométrica se realizó utilizando las técnicas de medición de uso internacional [16,17]. El peso de la gestante se midió en kilogramos con una báscula electrónica marca Tanita H327 de $0,1 \mathrm{~kg}$ de precisión y capacidad de $150 \mathrm{~kg}$. El peso del recién nacido se tomó en una pesa bebé de calzón marca Detecto con $0.05 \mathrm{~kg}$ de sensibilidad y $25 \mathrm{~kg}$ de capacidad y la longitud con un infantómetro marca Jadac con sensibilidad de $0.1 \mathrm{~cm}$ y capacidad de $100 \mathrm{~cm}$. Para determinar la ganancia de peso se utilizaron rangos de ganancia de acuerdo con el estado nutricional de la madre en el primer trimestre de gestación, según la propuesta de Atalah y colaboradores $[18,19]$. Los recién nacidos fueron evaluados en las salas de parto por profesionales de enfermería previamente estandarizados para tal fín. Los recién nacidos fueron clasificados de acuerdo a su peso así: > $4.000 \mathrm{~g}$ macrosómico, 3.999- $3.000 \mathrm{~g}$ adecuado, 2.999-2.500 g peso insuficiente y menor o igual a $2.500 \mathrm{~g}$ bajo peso [20].
Indicadores bioquímicos: la determinación de la ferritina sérica se valoró por un inmunoensayo de electroquimioluminiscencia (ECLIA) en un analizador automático (Roche Modular Analytics E170). El punto de corte para definir deficiencia fue $15 \mu \mathrm{g} / \mathrm{dl}$ [21]. El folato sérico se valoró por electroquimioluminiscencia, en un analizador automático (Roche Modular Analytics E170) y puesto que se desconocen valores de referencia para las gestantes, se emplearon aquellos recomendados por algunos investigadores para población sana no gestante [22]; la proteína $\mathrm{C}$ reactiva en suero se analizó por inmunoturbidimetria en equipo automático (Modular P800 de Roche) durante el embarazo se emplea como punto de corte 1,5 mg/dl [23]. La hemoglobina (Hb) se midió directamente utilizando el método de la cianometahemoglobina modificado, en equipo de lectura automática (Cell DYN 3700 de Abbott); el recuento total de glóbulos rojos (RBC), también se obtuvo directamente del equipo y a partir de los datos de distribución de su tamaño, se derivó el volumen corpuscular medio (MCV). $\mathrm{El}$ hematocrito se calculó a partir del RBC y el MCV; finalmente, la concentración de hemoglobina corpuscular media (MCHC), se obtuvo de la relación $\mathrm{Hb} / \mathrm{Hto} \mathrm{x}$ 100. La anemia durante la gestación, se determinó con los siguientes puntos de corte para $\mathrm{Hb}$ : primer y tercer trimestre $11,0 \mathrm{~g} / \mathrm{dl}$ y segundo trimestre $10,5 \mathrm{~g} / \mathrm{dl}$ [21].

El proyecto fue aprobado por el Comité de Bioética de la Universidad de Antioquia. Los aspectos éticos de esta investigación están basados en la resolución 008430 del 4 de octubre de 1993, por medio de la cual el Ministerio de Protección Social de la República de Colombia establece las normas científicas, técnicas y administrativas para desarrollar investigación en salud [24].

El procesamiento y análisis de los datos se hizo en el software SPSS, versión 14.0, excepto los datos de ingesta dietética que se procesaron utilizando software especializados tal y como se describió anteriormente en la sección de métodos. Las características económicas y sociodemográficas, así como el estado nutricional por indicadores antropométricos, bioquímicos y alimentarios, se describieron mediante proporciones, promedios, percentiles y desviación o error estándar. A las variables cuantitativas se les determinó la normalidad por medio de la pruebas Kolmogorov-Smirnov o Shapiro Will; para establecer cambios en las medias de concentración de indicadores bioquímicos y hematológicos entre los trimestres de gestación, se utilizó ANOVA de medidas repetidas y prueba de Friedman, además, se empleó Cochran para evaluar el cambio de variables cualitativas; las correlaciones, se establecieron con Pearson o Spearman según la distribución de la variables. Así mismo, como parte del análisis longitudinal, se efectuó 
el cálculo de las tasas de incidencia con sus respectivos intervalos de confianza del $95 \%$ y se utilizó como nivel de significación estadística para el control del error tipo $\mathrm{I}$, un valor alfa $=0.05$.

\section{RESULTADOS}

Determinantes demográficos y socioeconómicos de la seguridad alimentaria: Entre las participantes $20 \%$ tenían entre 16 y 18 años y $80 \%$ entre 19 y 40 años, de éstas 73,3\% residían en la zona urbana. En relación al nivel educativo se encontró que $32 \%$ tenía estudios completos o incompletos de básica primaria, $60 \%$ habían cursado algún grado de secundaria o la habían finalizado y sólo $8 \%$ tenía estudios superiores. La actividad predominante fue la de ama de casa $(81 \%)$.

El 70\% de los hogares tuvieron como jefe del hogar al esposo o compañero de la gestante, $7 \%$ a la madre de la gestante y $4 \%$ a la misma gestante. Respecto a los ingresos se encontró que $74,1 \%$ de los hogares percibieron menos de un salario mínimo mensual legal vigente -SMMLV- (USD \$234) y se encontró una diferencia estadística en el ingreso promedio entre las tres subregiones. La brecha entre los hogares de la subregión que presentó la media más alta y la subregión con el promedio más bajo fue de USD \$57. Los hogares invirtieron la mayor cantidad de sus recursos económicos en la compra de alimentos y en el pago de la vivienda y de los servicios públicos.

Los materiales predominantes en la construcción de las viviendas fueron el ladrillo y el cemento $65,7 \%$ y $64,8 \%$ respectivamente; sin embargo, una proporción considerable de las viviendas estaban construidas en madera (21\%) y poseían piso de tierra (20\%). De las viviendas el $74 \%$ tenía acueducto, pero de estas sólo el $6 \%$ tenían suministro de agua potable. En promedio, $70 \%$ de los hogares de las tres subregiones se percibieron en seguridad alimentaria, de los cuales la mayor proporción tenían como jefe de hogar al esposo o compañero de la gestante $(63,6 \%)$.

Evaluación de la ingesta dietética: El programa de alimentación complementaria logró el objetivo de incrementar en promedio el consumo de 300 calorías (Kcal) por día ( $\mathrm{p}<0,001)$. Antes de iniciar el programa de intervención, 91\% de las participantes estuvo a riesgo de deficiencia en la ingesta usual de calorías, deficiencia que se redujo a $81,6 \%$ en la evaluación realizada a las madres en el tercer trimestre. El consumo de proteínas y de grasas fue constante durante toda la gestación y estuvo por debajo de la cantidad recomendada.

En relación al consumo de vitaminas y minerales, se encontró disminución en el riesgo de deficiencia de los nutrientes suministrados a las madres por medio del complemento alimentario y del suplemento de micronutrientes. En todas las subregiones se presentó un incremento estadísticamente significativo en el consumo de vitamina A, C, y folatos, $(\mathrm{p}<0.001)$. En general la subregión Norte, tuvo la menor prevalencia de deficiencia en la ingesta usual de estos nutrientes. Es de anotar que la subregión Urabá en el tercer trimestre, tuvo un descenso en el consumo de folato y vitamina $\mathrm{C}$, lo cual posiblemente se debió a una menor ingesta del suplemento entregado.

Respecto a los minerales se destaca que en el primer trimestre $90,1 \%$ de las participantes presentó riesgo de deficiencia en la ingesta usual de cinc, la cual descendió al 10,6\% en el tercer trimestre. Con relación al calcio, el riesgo de deficiencia disminuyó de manera apreciable y pasó de $91,7 \%$ en el primer trimestre a $54,1 \%$ en el tercero; en cuanto al hierro, en el primer trimestre hubo una ingesta media de $12,5 \mathrm{mg}$, la cual a partir del segundo trimestre presentó un incremento significativo $(\mathrm{p}<0.001)$ alcanzando un promedio de 55,3 $\mathrm{mg}$.

$\mathrm{Al}$ analizar la ingesta de alimentos la dieta de las gestantes se caracterizó por ser monótona, baja en frutas, verduras, lácteos, carnes, grasas y alta en harinas. La subregión del Norte presentó el mayor número de madres que ingirió cinco comidas por día, lo cual coincide con un menor riesgo de deficiencia y un mayor riesgo de exceso en el consumo de energía.

El programa de complementación alimentaria y suplementación de micronutrientes, tuvo buena aceptación lo que contribuyó a la adherencia en el consumo de los productos por parte de las gestantes, dicho consumo fue mayor en los sitios donde se mantuvo el énfasis en la educación nutricional. En ocasiones los alimentos (leche MANA, galleta y bienestarina) se compartieron con el grupo familiar.

Evaluación antropométrica: Se encontró que $65,7 \%$ de las gestantes tenían un IMCG (índice de Masa Corporal Gestacional) adecuado, 27,8\% bajo y 9,5\% con sobrepeso en el primer trimestre. El IMCG promedio en el primer trimestre fue de $22,2 \mathrm{Kg} / \mathrm{m}^{2}$. Al comparar los valores de Índice de Masa Corporal (IMC) en cada uno de los trimestres de gestación se observaron cambios en la clasificación sin diferencias estadísticamente significativas. El bajo peso disminuyó progresivamente en la medida en que se desarrolló el programa, pasando de $27,8 \%$ en el primer trimestre, a $26,6 \%$ en el segundo y $20,3 \%$ en el tercero. En la región de Urabá el bajo peso se redujo de $44 \%$ en el primer trimestre a $29.5 \%$ en el tercer trimestre. (figura 2)

El 94\% de los recién nacidos tuvieron pesos superiores a los $2500 \mathrm{~g}$. Al relacionar el peso al nacer con el IMC materno se encontró que $68,5 \%$ de los niños con 
pesos entre 3.000 y $4.000 \mathrm{~g}$ eran de madres con IMC adecuado en el primer trimestre de la gestación. De las 22 gestantes que presentaron IMC bajo en el primer trimestre, dos tuvieron hijos con bajo peso y siete con peso insuficiente. El peso promedio de los niños al nacer fue de $3201 \mathrm{~g}$ con una variación de $\pm 629 \mathrm{~g}$.

Como se describe en la metodología, para determinar si las ganancias de peso de las madres fueron adecuadas a su estado nutricional pregestacional, se definieron rangos de ganancia de peso según la propuesta de Atalah y col $[18,19]$. De las 5 madres que tuvieron hijos con bajo peso, 3 tuvieron baja ganancia de peso. Al comparar la proporción de recién nacidos con bajo peso y peso insuficiente, entre las madres que alcanzaron una ganancia de peso adecuada a su estado nutricional pregestacional versus las que presentaron baja ganancia, no se hallaron diferencias significativas $(\mathrm{p}=0,099)$. Sin embargo, se evidenció mayor proporción de recién nacidos con peso mayor a $3.000 \mathrm{~g}$ en las madres con ganancias de peso adecuadas a su estado nutricional preconcepcional $(75,6 \%)(\mathrm{p}=0,031)($ tabla 1$)$.
Evaluación bioquímica del estado nutricional del hierro y folato: La figura 2 muestra los resultados de ferritina, $\mathrm{Hb}$ y de los índices eritrocitarios para el total de madres por trimestre de gestación. La mayoría de los indicadores disminuyeron en el segundo trimestre con relación al primero, y las diferencias fueron estadísticamente significativas excepto para la concentración de MCHC; al final de la gestación, la ferritina y la MCHC descendieron aún más con relación al segundo trimestre, pero este cambio no alcanzó a ser significativo. $\mathrm{La} \mathrm{Hb}$ y RBC aumentaron del segundo al tercer trimestre de gestación, sin embargo, la diferencia solo fue significativa para el RBC.

De acuerdo con los resultados anteriores, la prevalencia de ferropenia se incrementó en el embarazo y alcanzó a ser significativa entre el primer y tercer trimestre $(\mathrm{p}=0,000)$; los casos de anemia, cuatro en total, se diagnosticaron en madres adolescentes y que no tuvieron una ingesta regular del suplemento. Respecto a las maternas con ferropenia en algún trimestre de la gestación $(n=22)$, es importante destacar que sólo

\section{FIGURA 2}

Comparación del IMC gestacional entre primer, segundo y tercer trimestre.

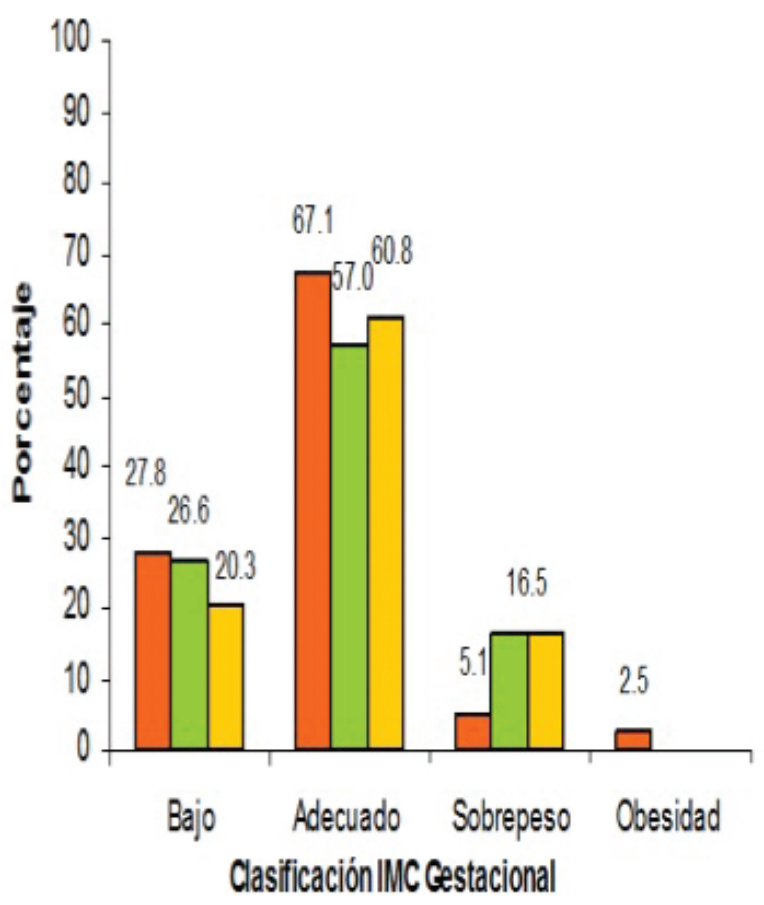

a 1 Trimestre $\quad$ Q2 Thimestre $\quad$ Q3Timestre 


\section{TABLA 1}

Clasificación del peso del niño al nacer vs clasificación final de la madre de acuerdo a su ganancia de peso total

\begin{tabular}{lcccccrcr}
$\begin{array}{l}\text { Clasificación } \\
\text { Peso Niño }\end{array}$ & \multicolumn{4}{c}{$\begin{array}{c}\text { Ganancia total de peso de la madre } \\
\text { Inadecuada } \\
\text { por déficit }\end{array}$} & $\begin{array}{c}\text { Inadecuada } \\
\text { por exceso }\end{array}$ & Adecuada & & Total \\
& n & $\%$ & n & $\%$ & n & $\%$ & n & $\%$ \\
\hline Bajo Peso $<2500$ & 3 & 10,0 & 1 & 14,3 & 1 & 2,4 & 5 & 6,4 \\
Insuficiente $<3.000->2.500$ & 9 & 30,0 & 3 & 42,8 & 8 & 19,5 & 20 & 25,6 \\
Adecuado $>3.000-4.000$ & 17 & 56,7 & 2 & 28,6 & 31 & 75,6 & 50 & 64,2 \\
Macrosómico $>4.000$ & 1 & 3,3 & 1 & 14,3 & 1 & 2,5 & 3 & 3,8 \\
Total & 30 & 100 & 7 & 100 & 41 & 100 & 78 & 100 \\
\hline
\end{tabular}

\section{FIGURA 3}

Concentración de variables bioquímicas e índices eritocritarios por trimestre de gestación.

Subregión Bajo Cauca.

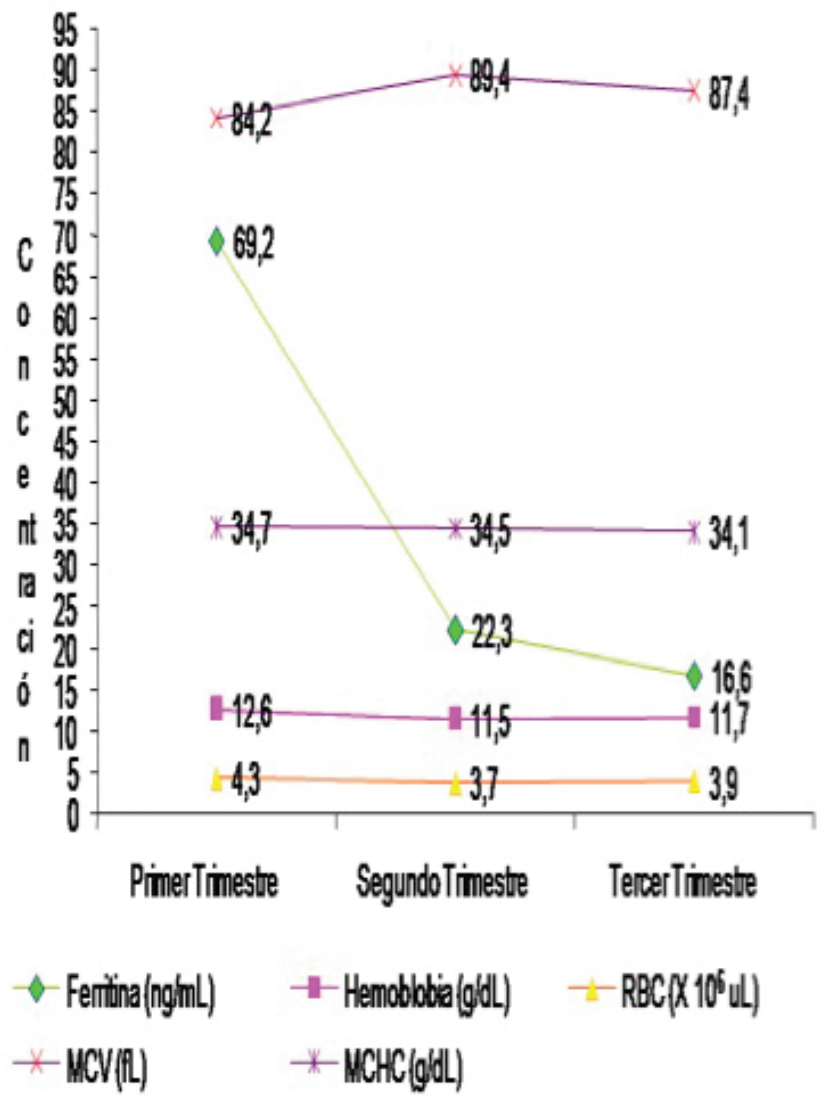


$3(14 \%)$ de ellas presentó anemia, lo que significa que en $86 \%$ de madres con deficiencia de hierro, se previno esta patología. (figura 3)

En el segundo trimestre, se observó una prevalencia de $61,5 \%$ de las gestantes con promedio bajo de glóbulos rojos, lo que fue significativo ( $\mathrm{p}=0,000)$ con relación al inicio de la gestación, donde ninguna madre se clasificó en esta categoría, sin embargo, el porcentaje disminuyó en el tercer trimestre a 30,8\%. Con relación al tamaño del glóbulo rojo, ninguna madre presentó macrocitosis no obstante, la microcitosis fue del $23,1 \%$ en las gestantes al iniciar el estudio, con una proporción de adolescentes tres veces mayor en comparación con las mujeres adultas; este porcentaje disminuyó a 3,8\% en el segundo trimestre y se mantuvo al final de la gestación, el cambio fue estadísticamente significativo $(\mathrm{p}=0,028)$.

En cuanto al estado nutricional del folato, se observó un aumento en la concentración sérica de la vitamina en el segundo y tercer trimestre de embarazo, con relación al primero y los cambios fueron significativos $(\mathrm{p}=0,005$ y $\mathrm{p}=0,032$, respectivamente); en concordancia con este incremento, disminuyó la proporción de madres a riesgo de deficiencia de esta vitamina de $30,8 \%$ en el primer trimestre de gestación, a $11,5 \%$ en el segundo, efecto generado por el suplemento de ácido fólico; al final del embarazo la prevalencia del riesgo fue de $8 \%$. Se destaca el aumento en la proporción de mujeres con balance positivo de folato en el segundo trimestre $50 \%$ con respecto al primero $(7,7 \%)$, pese a que los cambios fisiológicos maternos también parecen incidir en el descenso plasmático de esta vitamina (figura 4).

\section{DISCUSIÓN}

Un $74.1 \%$ de los hogares tuvieron ingresos inferiores a un SMMLV, superando el promedio reportado por el Perfil Alimentario y Nutricional del departamento Antioquia realizado en el 2004 y que mostró ingresos inferíos al SMMLV en el $60,8 \%$ de la población [25]. El $48,3 \%$ de las familias invirtieron la mitad de sus limitados ingresos en la compra de alimentos, monto similar al

\section{FIGURA 4}

Distribución de las madres por categorias de folato sérico según trimestre de gestación. Subregión Bajo Cauca.

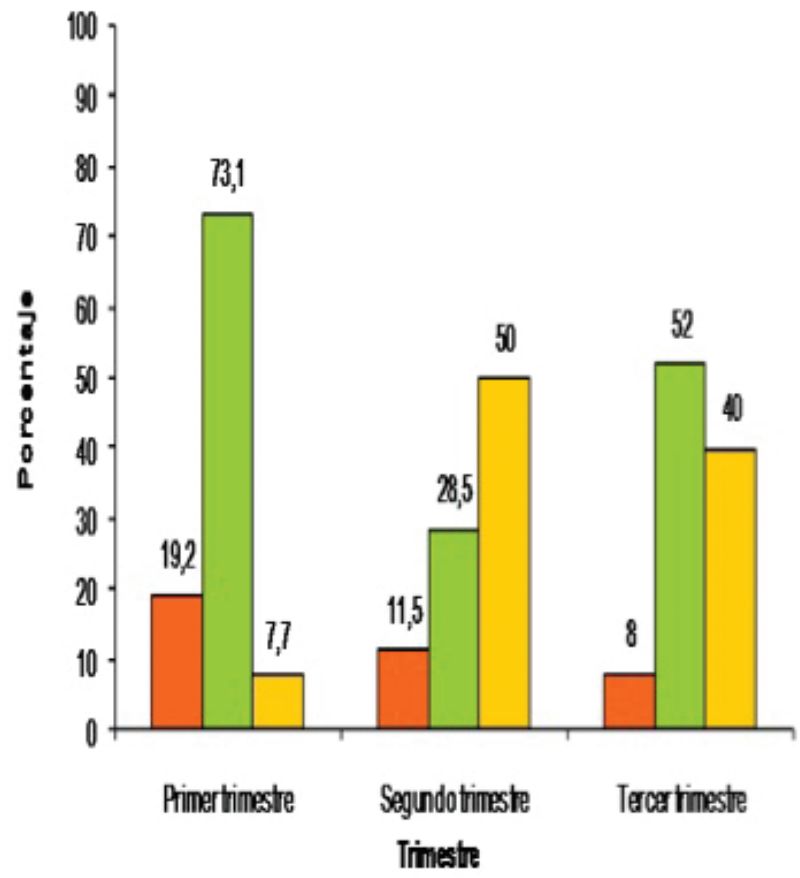

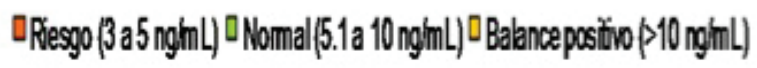


que se registró en el Departamento de Antioquia (55\%) [25] y que está lejos del porcentaje establecido para que un hogar prototipo cubra las necesidades mínimas de energía, el cual corresponde al 107\% del SMMLV de 2004 [25]. El porcentaje invertido en la compra de alimentos fue coherente con el que reportan otros autores, quienes además consideran que las personas priorizan esta necesidad básica frente a la inversión en otros rubros $[26,27]$.

El grado de escolaridad y la autonomía de la mujer para tomar decisiones son aspectos que se refuerzan mutuamente y que son determinantes en la supervivencia de los hijos, en este estudio un tercio de las gestantes solo alcanzó la básico primaria y solo un $8 \%$ estudios universitarios, lo que cobra importancia ya que la Encuesta Nacional de Demografía y Salud del año 2005, evidenció que la probabilidad de muerte de un niño es tres veces mayor en madres sin educación con respecto a las que tienen educación superior [3].

Diferentes estudios han mostrado que los hogares con jefatura femenina son más pobres que los encabezados por el hombre; por el contrario, la mayoría de los hogares con jefatura masculina, posibilitan que la mujer se encargue del trabajo del hogar y del cuidado de los hijos, según la CEPAL esto ocurre en el $88 \%$ de los hogares de América Latina [28]. En ésta investigación se observó que el porcentaje de hogares con jefatura femenina $(11 \%)$ es más bajo comparado con la cifra nacional (30\%) [3], sin embargo, es una proporción preocupante por la implicaciones que esto general para el binomio madre-hijo y para la seguridad alimentaria del resto de los miembros del hogar.

Respecto a la ingesta dietética, se encontró en el primer trimestre de gestación una alta prevalencia de riesgo de deficiencia en la ingesta usual de energía. El programa de complementación alimentaria logró el objetivo de incrementar en promedio 300 Calorías/día ( $\mathrm{p}=0.001)$ y disminuyó la proporción de madres con déficit energético en todas las subregiones, sin embargo la prevalencia de riesgo continuó siendo alta debido a que las necesidades energéticas incrementan en cada uno de los trimestres de gestación y la población intervenida tenia limitados recursos económicos, bajo nivel educativo y precarias condiciones sociales que limitan el acceso a los alimentos.

Estas carencias nutricionales durante el embarazo, están asociadas con inadecuado desarrollo del feto y mayor predisposición a enfermedades crónicas en la edad adulta $[29,30]$. La hipótesis de Barker propone que las adaptaciones ocasionadas por el déficit intrauterino cambian permanentemente las estructuras, la fisiología y el metabolismo ocasionando trastornos en etapas pos- teriores [31], además una ganancia insuficiente de peso durante la gestación es un factor de riesgo para bajo peso al nacer, parto prematuro y defectos congénitos [32].

En el otro extremo del riesgo se encuentran las mujeres con exceso en la ingesta de energía, situación que fue más prevalente en la subregión Norte y donde además hubo el mayor sobrepeso evaluado por IMC (23\%), lo que predispone a diabetes, cesárea, macrosomia fetal, defectos congénitos y obesidad en la vida posterior $[33,34]$. En el grupo de estudio convergen un inadecuado consumo de proteínas y un insuficiente respaldo calórico, condiciones que limitan la formación de los tejidos, ya que las proteínas cumplen la prioridad metabólica de producir energía.

Las vitaminas y minerales también juegan un papel trascendental en la formación de las estructuras del feto, desafortunadamente en las poblaciones pobres donde la dieta es poco variada es aun más difícil alcanzar las recomendaciones [31]. En este estudio, se observó que las mayores deficiencias de micronutrientes fueron las de calcio, folatos, hierro y cinc, situación que coincide con otras observaciones realizadas en Antioquia [35], en otras etnias y países $[32,36]$, estas deficiencias disminuyeron con el consumo del complemento alimentario y el suplemento de micronutrientes.

Otro aspecto a considerar al hacer referencia al estado nutricional materno, es el peso pregestacional y la ganancia de peso materno, ya que estos pueden ser determinantes del peso al nacer [37]. La ganancia de peso materno debe ser acorde con el estado nutricional de la madre, una mujer enflaquecida requerirá de una mayor ganancia de peso que una mujer con estado nutricional normal o con exceso. En este estudio se encontró que la mayor proporción de niños con pesos superiores a $3.000 \mathrm{~g}$ fueron los de las madres con adecuado peso pregestacional y con adecuada ganancia de peso durante la gestación.

Un IMC pregestacional bajo como indicador de déficit crónico de energía es de trascendental importancia en la gestación, mucho más si se trata de gestantes adolescentes que aún no han terminado su período de crecimiento y desarrollo por sus mayores demandas energéticas [38,39]. Varios estudios resaltan cómo el estado nutricional deficiente antes de la gestación influye de manera negativa tanto en la madre como en su futuro bebé $[5,40]$ y puede desencadenar retardo en el crecimiento intrauterino [41].

Durante la segunda mitad del embarazo la ganancia inadecuada de peso predice resultados perinatales adversos [37]. Una baja ganancia puede ser consecuencia de una ingesta inapropiada durante el embarazo y de necesidades básicas insatisfechas, que pueden desen- 
cadenar producción local de prostaglandinas y parto prematuro [40,42-44].

Los requerimientos de hierro y folato son especialmente altos en la gestación por lo que se recomienda la ingesta diaria de un suplemento para controlar la deficiencia de hierro, prevenir la anemia y contribuir a la salud y el bienestar materno fetal [45-47], las carencias de estos micronutrientes contribuyen al mal resultado obstétrico [48-51]. El presente estudio evaluó el estado de hierro y folato antes y durante un programa de intervención nutricional y encontró que el $50 \%$ de las gestantes en el segundo trimestre tuvo reservas de hierro que indicaron deficiencia $(<24 \mathrm{ng} / \mathrm{mL})$ y al final del embarazo, estuvieron cercanas a la depleción $(<15 \mathrm{ng} /$ $\mathrm{mL})[17,52]$. La Hb materna al inicio fue de $12,6 \mathrm{~g} / \mathrm{dL}$, la cual se esperaría hubiese sido más alta por el ahorro de hierro debido al cese de la menstruación.

La máxima utilización de las reservas de hierro para la expansión del volumen eritrocitario, los requerimientos placentarios y el crecimiento fetal, se reflejó en el descenso considerable de la ferritina y contribuyó al aumento de la ferropenia, especialmente en aquellas madres que tuvieron en el primer trimestre concentraciones cercanas a la deficiencia. Estos resultados coinciden con los obtenidos por Lee y col en mujeres coreanas, donde tanto el hierro sérico como la ferritina descendieron a lo largo de la gestación, pese a que las maternas tomaron un suplemento diario con $40 \mathrm{mg}$ de hierro elemental [52]. En nuestro caso, las madres recibieron una dosis diaria de fumarato ferroso con $60 \mathrm{mg}$ de hierro sin embargo, algunas adolescentes tuvieron una ingesta irregular lo que se reflejó en un aumento de la ferropenia en el segundo trimestre y de la anemia a lo largo de la gestación; es importante anotar, que no se presentó ningún caso de anemia severa $(\mathrm{Hb}<7 \mathrm{~g} / \mathrm{dL}) \mathrm{o}$ moderada (7-8,9 g/dL) [53]; todos los casos, fueron leves (9-10,9 g/dL); al respecto, es probable que las mayores demandas de hierro en las adolescentes por su propio crecimiento y las necesidades adicionales del embarazo, hayan contribuido a una máxima utilización de las reservas que no fueron suficientes para una adecuada síntesis de hemoglobina.

Aun cuando en este estudio la prevalencia de anemia aumentó en el segundo y tercer trimestre, la diferencia no fue significativa con relación al comienzo del embarazo, sin embargo, los casos se valoraron por su importancia clínica; en otros estudios, se reportan frecuencias mucho más altas en mujeres que no ingieren suplementos de hierro diariamente, o lo hacen de manera irregular durante el embarazo [54] por lo que es posible proponer que el seguimiento de la madre y el programa de educación nutricional que se implementó en esta investigación, contribuyó a tener menores prevalencias de anemia.

Se conoce que en ausencia de un tratamiento con suplementos de hierro, el $80 \%$ de las maternas puede alcanzar una concentración de $\mathrm{Hb}$ inferior a $11 \mathrm{~g} / \mathrm{dl}$ al final del embarazo [54] sin embargo en el presente estudio fue de 11,7 g/dl. Pese al descenso de la $\mathrm{Hb}$ y el RBC en el segundo trimestre, el tamaño y la concentración de $\mathrm{Hb}$ promedio de los glóbulos rojos fue normal y de seis casos de microcitosis que se diagnosticaron al inicio del embarazo, cinco se resolvieron al final y una gestante que presentó hipocromía en el primer trimestre, también mejoró al término del embarazo. Estos resultados ilustran el reporte de la Organización Mundial de la Salud - OMS-, donde el MCV se considera uno de los más importantes indicadores de impacto, de programas de intervención para controlar la deficiencia de hierro [55].

En nuestro estudio, encontramos correlación positiva y estadísticamente significativa entre la hemoglobina materna en segundo trimestre y el peso al nacer. Todo lo anterior enfatiza la importancia de mantener a lo largo de la gestación, una adecuada concentración de hemoglobina y evitar su disminución más allá de lo que podría esperarse como fisiológico y además evitar la anemia, con el fin de contribuir a un mejor resultado obstétrico [48,56,57].

Mejorar la ingesta de folatos en la dieta con la administración de un suplemento, también se considera necesario [58,59]; al respecto, se observó que al inicio del estudio cuando las madres no estaban recibiendo el suplemento, hubo mayor prevalencia de riesgo de deficiencia de folato sérico. Varias investigaciones han demostrado los beneficios del suplemento y la fortificación de alimentos con ácido fólico, sobre el estado nutricional materno fetal $[60,61]$. En esta investigación, los resultados demuestran claramente un mejoramiento en la concentración materna de folato sérico en el segundo y tercer trimestre que fue significativa con relación al primero, donde el promedio de folato fue mas bajo. Vale la pena resaltar la correlación positiva que se halló entre la ingesta de folatos y la concentración sérica de la vitamina en el tercer trimestre.

Por todo lo anterior y dado que las condiciones socioeconómicas de la población se mantuvieron durante la investigación se concluye que el programa desarrollado, los productos entregados y la educación nutricional tuvieron un impacto positivo y significativo en el estado nutricional de las madres, aspecto relevante ya que como se ha mencionado para cada uno de los indicadores del estado nutricional materno, existe suficiente evidencia científica que permite concluir que la nutrición juega un papel de trascendental importancia en el desarrollo del feto y la salud materna y más tarde en la salud y 
capacidad de trabajo del ser humano.

Para romper el círculo de la desnutrición madrehijo se requiere de estrategias de detección temprana de riesgos, vigilancia nutricional y atención en salud con calidad y oportunidad que permitan intervenir de manera oportuna las gestantes con vulnerabilidad alimentaria y riesgos nutricionales.

\section{RESUMEN}

Objetivo: Evaluar el estado nutricional de un grupo de gestantes y sus recién nacidos participantes del programa MANA para la vida. Método: Estudio descriptivo, longitudinal, prospectivo con seguimiento de la cohorte antes y después de una intervención. Resultados: el 53\% de los hogares se percibieron en inseguridad alimentaria. La ingesta de nutrientes tuvo un incremento significativo y se redujo la prevalencia del riesgo de deficiencia de algunos micronutrientes. El bajo peso gestacional disminuyó de $27,8 \%$ en el primer trimestre a $20,3 \%$ en el tercero. El $94 \%$ de los recién nacidos tuvieron un peso superior a $2500 \mathrm{~g}$. La anemia se previno en el $86 \%$ de las madres con deficiencia de hierro y se disminuyó la proporción a riesgo de deficiencia de folato. Conclusión: Dado las condiciones socioeconómicas de la población, se concluye que el programa desarrollado y los productos entregados tuvieron un impacto positivo y significativo en el estado nutricional de las madres.

Palabras clave: gestación; complementación alimentaria; suplemento de micronutrientes; educación nutricional; estado nutricional.

Dirigir la correspondencia a:

Profesora

Sandra Restrepo M.

Escuela de Nutrición

Universidad de Antioquia

Colombia

Teléfono: 4259227 - 2199200

E-mail: sanres@pijaos.udea.edu.co

Agradecimientos: El grupo de investigación agradece a la Gobernación de Antioquia y a la Gerencia Departamental de Seguridad Alimentaria y Nutricional por la iniciativa y el aporte financiero para el desarrollo del programa de intervención y del proyecto de investigación. A la Dirección de Salud Pública del Servicio Seccional de Salud de Antioquia por su porte con el suplemento de micronutrientes para las gestantes. A las Empresas Sociales del Estado, sus gerentes y funcionarios que hicieron posible cada una de las actividades y de manera especial a las gestantes, sujetos centrales de este proyecto.

\section{BIBLIOGRAFÍA}

1. Ministerio de Protección Social. Situación de salud en Colombia: Indicadores básicos 2007.http://minproteccionsocial.gov.co/VBeContent/NewsDetail. asp? ID $=15895 \&$ IDCompany $=3$. [Fecha de acceso abril 15 de 2008].

2. Dirección seccional de salud de Antioquia. Número y tasa de las muertes maternas 2006.http://www. dssa.gov.co/dowload/archivoseventos_2007/ MorInfantilMaternaPerinatal1995-2006ver2.XLS. [Fecha de acceso abril 15 de 2008].

3. Profamilia. Encuesta Nacional de Demografía y Salud: Salud sexual y reproductiva, Bogotá 2005. http://www.profamilia.org.co/encuestas/01encuest as/2005resultadosgenerales.htm. [Fecha de acceso Marzo 6 de 2007]

4. López J, Lugones M, Valdespino L, Virilla J. Algunos factores maternos relacionados con el bajo peso al nacer. Rev Cubana Obstet Ginecol. 2004; 30(1):s/p.

5. Balestena J, Suárez C, Balestena S. Valoración nutricional de la gestante. Rev Cub Obstet y Ginecol. 2001;27(2):165-71.

6. Ministerio de Salud. Resolución 00412 de febrero 25 de 2000. Bogota URL www.minproteccionsocial. gov.co. [Fecha de acceso Marzo 3 de 2007.

7. Berrio K. La Salud en Antioquia, una mirada desde la administración. En: Departamento de Antioquia, Dirección Seccional de Salud de Antioquia. La salud de las madres en Antioquia: un reto, un derecho, un compromiso. Medellín: DSSA; 2007. 45-55. Libro disponible en internet: URL: http://www. nacer.udea.edu.co/pdf/libros/libro3/03.pdf. [Fecha de acceso 13 de noviembre de 2009]

8. Londoño J. Metodología de la Investigación Epidemiológica. Medellín: Universidad de Antioquia; 1995;pp 25-39.

9. Navarro H. Manual para la evaluación de impacto de proyectos y programas de lucha contra la pobreza. Santiago: CEPAL; 2005 [acceso enero 2009] Serie Manuales, No. 41. Disponible en: http://www.eclac. org/cgi-bin/getProd.asp?xml=/publicaciones/).

10. Alvarez M, Estrada A, Montoya E, Melgar H. Validación de la escala de la seguridad alimentaria doméstica en Antioquia, Colombia. Salud Púb Méx. 2006;48(6):474-81

11. Manjarrés L, Correa J. Software para la Evaluación de la Ingesta dietética Evindi. Medellín: Escuela de Nutrición y Dietética, Universidad de Antioquia; 2006.

12. Instituto Colombiano de Bienestar Familiar. Tabla de composición de alimentos colombianos. Bogotá: 
Talleres de sesión de Publicaciones; 1988;

13. FAO. Latinfoods 2002. http://www.rlc.fao.org/ bases/alimento/default.htm. [Fecha de acceso 22 de marzo de 2004].

14. USDA. Hand Book 8. http://www.hoptechno.com/ nightcrew/sante4me/usda19datashape.cfm. [Fecha de acceso 17 de febrero de 2004].

15. Ministry of Health, New Zealand National Health and Medical Research Council. (NHMRC). Evidence appendix for nutrient reference values for Australia and New Zealand including Recommended Dietary Intakes. [Documento en internet] Disponible en URL: http://www.ag.gov.au/portal/ govgazonline.nsf/0/B1358DE3EE607F08CA256 F7000278423/\$file/S527. [Fecha de acceso marzo de 2005].

16. Jackson A, Pollock M. Practical assessment of body composition. The Physician and Sport Medicine. 1985;13(5):76-89

17. Harrison G, Buskirk E, Carter J, Johnston F, Lohman T, Pollock M, et al. Skinfold Thicknesses and Measurement Technique. En: Lohman T, Roche A, Martorell R, ed. Antropometric Standardization Reference Manual. Champaign IL: Human Kinetics Publishers; 1988; 55-70.

18. Atalah E, Castillo C, Castro R, Aldea A. Propuesta de un nuevo estándar de evaluación nutricional de embarazadas. Rev Med Chile.1997;125:1429-36

19. Uauy R, Athalah E, Barrera C, Behenke E. Alimentación y nutrición durante el embarazo. En: Uauy R, Athalah E, Barrera C, Behenke E, ed. Guías de alimentación para la mujer. Chile: Universidad de Chile; 2001;53-74.

20. Restrepo M. Evaluación del crecimiento intrauterino y del recién nacido. En: Restrepo M, ed. Estado nutricional y crecimiento físic 1ed. Medellín: Universidad de Antioquia; 2000; p.p.182.

21. UNICEF, UNU, OMS. Iron Deficiency Anaemia: Assessment, Prevention and Control, a guide for programme managers. Washington: OMS; 2001;99101.

22. Lee R, Nieman D. Biochemical Assessment of Nutritional Status. En: Lee R. Nieman D, ed. Nutritional Assessment 3 ed. New York: McGraw-Hill; 2003;325-327.

23. Maternal Physiology. En: Cunningham F, Leveno K, Bloom S, Hauth J, Gilstrap III L, Wenstrom K, ed. Williams Obstetrics 22 ed. New York: McGrawHill; 2005; p.p.131.

24. Congreso de la República de Colombia. Resolución $\mathrm{N}^{\circ} 008430$ del 4 de octubre de 1993: Por la cual se establecen las normas científicas, técnicas y admin- istrativas para la investigación en salud. Bogotá: Ministerio de salud; 1993.

25. Álvarez M, Roldán P, Benjumea M, Martinez M, Maya M, Montoya I. Perfil Alimentario y Nutricional de los Hogares de Antioquia. Medellín: Gobernación de Antioquia, Universidad de Antioquia; 2003; 45-63.

26. Figueroa D. Acceso a los alimentos como factor determinante de la seguridad alimentaria y nutricional y sus representaciones en Brasil. Rev Costarric Salud pública. 2005;14(27):s/p.

27. Garay L, Rodríguez A. Seguridad Alimentaria más allá del derecho a no padecer hambre. En: Garay L, Rodríguez A, ed. Planeta Paz: Diálogo pendiente 1ed. Bogotá: Planeta paz; 2005; p.p.219-279.

28. CEPAL. Serie Mujer y desarrollo: Entender la pobreza desde la perspectiva de género, 2004. www.cepal.org [Fecha acceso 20 de septiembre de 2005].

29. Podja J, Kelly L. Long - term consequences of low birth weight: The fetal origenes of disase hyphotesis. Low birthweight. Nutrition Policy Paper. En: ACC/SCN, WHO; 2000.

30. Barker D. Maternal nutrition, fetal nutrition, and disease in later life. Nutrition. 1997;13:807 - .

31. Pineda D, Martín I, Macias M, Monterrey P, Pita G, Serrano G. Ingesta de macronutrientes y vitaminas en embarazadas durante un año. Rev Cubana Salud Pública 2003; 29(03): 220 - 227.

32. Watts V, Rockett H, Baer H, Leppert J, Coltiz G. Assessing Diet Quality in Population of Low-Income Pregnant Women: A comparison Between Native Americans and Whites. Matern Child Health J 2007; 11: 127 - 36.

33. Galtier-Dereure F, Boegner C, Bringer J. Obesity and Pregnancy complications and cost. Am J Clin Nutr 2000; 71: 1242S - 1248S.

34. Cedergren M. Effects of gestational weight gain and body mass index on obstetric outcome in Sweden. Int J Gynecol Obstet 2006; 93:269 - 74

35. Manjarrés L, Restrepo S, Arboleda R. Perfil alimentario y hematológico. Estudio en mujeres gestantes asistentes al programa prenatal de la Empresa Social del Estado Hospital Gilberto Mejía Mejía. Municipio de Rionegro-Antioquia (1998-1999). Perspectivas en Nutrición Humana. 2001;4:11 -26.

36. Peña E, Sánchez A, Portillo Z. Evaluación dietética de adolescentes embarazadas durante el primer, segundo y tercer trimestre. ALAN. 2003; 53(2): 133-140.

37. Ehrenbern H, Dierker L, Milluzzi C, Mercer B. Low maternal weight failure to thrive in pregnancy and 
adverse pregnancy outcomes. Am J Obstet Gynecol 2003;18(6): 1726-1730

38. King J, Weininger J. Gestación y Lactancia. En: OPS, ed. Conocimientos actuales sobre nutrición 6ed. Washington: OPS, ILSI; 1991;394

39. Shetty P, James W. Health and BMI. En: Shetty $\mathrm{P}$, James W, ed. Body mass index, a measure of chronic energy deficiency in adults. Roma: FAO; 1994;11-16.

40. Grandi C. Relación entre la antropometría materna y la ganancia de peso gestacional con el peso de nacimiento, y riesgos de peso bajo al nacer, pequeño para la edad gestacional y prematurez en una población urbana de Buenos Aires. ALAN. 2003; 53(4):369-375.

41. Doherty DA, Magann EF, Francis J, Morrison JC, Newnham JP. Pre-pregnancy body mass index and pregnancy outcomes. Int J Gynecol Obstet 2006; 95: 242-47

42. Calle A. Crecimiento fetal: su evaluación clínica. En: Gestación y lactancia las deficiencias nutricionales de la mujer andina ecuatoriana 1 ed. Quito:PROPRUMED; 2004; 159-165

43. Hosseini M, Nastarán J. Relationship betwen pregnancy outcome and maternal BMI and weight gain. International Congress Series 12712. 2004:380-383. http://www.sciencedirect.com/science. [Fecha de consulta: 2 de septiembre 2007]

44. Merlino A, Laffineuse L, Collin M, Mercer B. Impact of weight loss between pregnancies on recurrent preterm birth. Am J Obstet Gynecol 2006; 195:818-21.

45. Beard J. Effectiveness and strategies of iron supplementation during pregnancy. Am J Clin Nutr. 2000;71(suppl):1288S-94S.

46. Parra B, Manjarrés L, Gómez A, Alzate D, Jaramillo M. Evaluación de la educación nutricional y un suplemento para prevenir la anemia durante la gestación. Biomédica 2005;25: 211-19.

47. Acuña J, Yoon P, Erickson J. La prevención de los defectos del tubo neural con ácido fólico. Estados Unidos: Organización Panamericana de la Salud -OPS-, Centro para la prevención y el control de Enfermedades (CDC); 1999;1-13.

48. Levy A, Fraser D, Katz M, Mazor M and Sheiner E. Maternal anemia during pregnancy is an independent risk factor for low birthweight and preterm delivery. Eur J Obstet Gynecol Reprod Biol 2005;122: 182-86.

49. Preziosi P, Prual A, Galán P, Daouda H, Hamidov B, et al. Effect of iron supplementation on iron status of pregnant women: Consequences for newborns. Am J Cl Nut 1997; 66: 1178- 82.

50. Caudill M, Cruz A, Gregory J, Hutston A, Bailey L. Folate Status Response to Controlled Folate Intake in Pregnant Women. J Nutr 1997;127:2363 -70.

51. American D, Association. Position of the American Dietetic Association: Nutrition and lifestyle for a healthy pregnancy outcome. J Am Diet Assoc 2002; 102 (10): 1479 - 90.

52. Lee JI, Kang S A, Kim S-K and Lim H-S. A cross sectional study of maternal iron status of Korean women during pregnancy. Nutr Res. 2002; 22: 1377-88.

53. Akesson A, Bjellerup P, Berglund M, Bremme K, Vahter M. Serum transferrin receptor: a specific marker of iron deficiency in pregnancy. Am J Clin Nutr 1998; 68: 1241-46.

54. Perry K. Alteraciones de los eritrocitos. En: Fridman J, Klajn D, Meeroff N, Patrone U, ed. Tratamiento de las complicaciones clínicas del embarazo 3ed. Argentina: Editorial Médica Panamericana SA; 2000;1354-62

55. OMS, CDC. Assessing the Iron Status of populations. Report of a Joint World Health Organization/ Centres for Disease Control and Prevention. Switzerland: WHO Press; 2005; p.p.8-13

56. Ren A, Wang J, Ye R, Li S, Liu J, Li Z. Low firsttrimester haemoglobin and low birth weight, preterm birth and small for gestational age newborns. Int J Gynecol Obstet 2007;98: 124-28.

57. Little M, DPHIL, Brocard P, MPHIL, Elliott P, Steer P. Hemoglobin concentration in pregnancy and perinatal mortality: A London-based cohort study. Am J Obstet Gynecol 2005;193: 220-6.

58. Van IA, Ocké MC, Straatman H, Zielhuis GA, Merkus HM, Steegers RP. Periconceptional folate intake by supplement and food reduces the risk of nonsyndromic cleft lip with or without cleft palate. Prev Med 2004;39: 689-94

59. Siega-Riz A, Savitz D, Zeisel S, Thorp J, Herring A. Second trimester folate status and preterm birth. Am J Obstet Gynecol 2004; 191:1851-57.

60. Eichholzer M, Tönz O, Zimmermann R. Folic acid: a public-health challenge. Lancet 2006; 367: 1352-61

61. StarK K, Pawlosky R, Beblo S, Murthy M, Flanagan $\mathrm{V}$, Janisse J, et al. Status of plasma folate after folic acid fortification of the food supply in pregnant African American women and the influences of diet, smoking, and alcohol consumption. J Clin Nutr 2005;81:669-77. 